\title{
Positioning Technology for Firefighters' Emergency Rescue Based on CSS
}

\author{
Gaoping Liü, a , Huanhuan Tie ${ }^{2, b}$, Chengrong Dai ${ }^{3, \mathrm{c}}$ \\ ${ }^{1}$ EDA Key Laboratory, Zhejiang Wanli University, Ningbo, 315100, China \\ ${ }^{2}$ EDA Key Laboratory, Zhejiang Wanli University, Ningbo, 315100, China \\ ${ }^{3}$ EDA Key Laboratory, Zhejiang Wanli University, Ningbo, 315100, China \\ aemail: 0574119@163.com, bemail: 634772859@qq.com, cemail: ddccrr112233@sina.com
}

Keywords: Chirp Spread Spectrum; Fire Rescue; 3D Positioning; Kalman Filter

\begin{abstract}
This paper researches a 3D positioning scheme for emergency rescue of trapped firefighters at the fire scene. This scheme adopts Chirp Spread Spectrum (CSS) and its particular Symmetric Double-Sided Two-Way Ranging (SDS-TWR). Combining BMP085 pressure sensor to measure altitude, it can implement 3D positioning of firefighters. Due to the complex environment of the fire scene would cause multipath effect when Chirp signal being transmitted, this scheme uses Kalman filtering method to filter out mutative coordinate value, thus the positioning error can be controlled within $1 \mathrm{~m}$.
\end{abstract}

\section{Introduction}

Due to the lack of awareness about fires, there are more and more news about the sacrifice of young firefighters. Since most fires have occurred in indoor, GPS satellite signal is easily obscured, while CSS technology can well improve indoor positioning accuracy. CSS is a communication technology which can realize spread spectrum by chirp signal. CSS can increase the signal transmission bandwidth by modulating the carrier realizing pulse compression when receiving, which has advantages of strong decay resistance ability, far transmission distance, low consumed power and so on [1]-[3]. CSS with its particular SDS-TWR, a kind of ranging method based on Time of Arrival (TOA), can calculate the distance between the Anchor and the positioning target according to the transmission time of wireless signal from the transmitter to the receiver, and get the positioning of the firefighters through a certain localization algorithm, which is in favor of emergency rescue of trapped firefighters.

\section{D Localization Algorithm and Error Compensation}

\section{System Architecture.}

In this paper, the emergency rescue positioning system is set up under the emergency situations such as fires; generally consist of the Tag, the Anchors and the monitoring terminal (PAD, PC or laptop).

Tag is the lowest level of the whole system responsible for measuring the distance between the Anchor, and sending positioning packets to all Anchors in the form of broadcast packets. Tags are installed on the mobile nodes (such as firefighters), and sends location to monitor terminal within the prescribed time, which realizes the real time positioning of Tag.

The block diagram of the internal structure about a typical Tag is shown in Fig.1. Among it, the air pressure sensor is used to measure the altitude of the Tag. Recently there are a lot of application researches using air pressure sensor for height measurement, such as [4-6], so this paper do not give more details.

All Anchors, which position are fixed, are regarded as the known coordinates positioning node. They are used to receive the positioning packet sent by the Tag, and calculate the distance between the Anchor and the Tags through CSS ranging principle. The number of the Anchors set by the scene of the emergency situation can be 2,3 or 4 . All Anchors is generally installed at the 
emergency scene temporarily (such as buildings, warehouses, etc), and the coordinates is set based on the location of the Anchors. One of the Anchors, connected to the monitoring terminal, outputs the received data to the monitoring terminal. The hardware block diagram of the Anchor is similar basically to Tags. Monitor plays a role of receiving the location and environment information sent by Tags, calculating the coordinates of the Tag with the 3D positioning algorithm below 1.2, filtering and correcting the data output by the Anchor. On the graphical interface of positioning software Monitor shows 3D position of the Anchors and the Tags in real time.

\section{D Localization Algorithms.}

Having known all the distance between the Anchors and Tags, and their relative height to the ground, you can get the moving Tag position through the geometric calculation.

Assumed that $z_{i}$ is the height of the Anchor $i(i \in[1, \mathrm{~N}]$, where $\mathrm{N}$ is the number of Anchors) to the ground, that is $z$ coordinate of the Anchor $i$. The altitude of Tags and Anchor $i$ is obtained by air pressure - high conversion [4]-[6], that is $h$, hi, respectively, thus the Tag z coordinate calculation formula is as follows:

$$
z=\left|h-h_{i}\right|+z_{i}
$$

Due to the Anchor is not strictly in the same horizontal plane, you need to project Anchors and Tags to the horizontal plane. The actual distance between the Tag and the Anchor is $r_{i}$, thus $r_{i}$ in the projection of the ground is:

$$
d_{i}=\sqrt{r_{i}^{2}-\left(z_{i}-z\right)^{2}}
$$

(1) When $\mathrm{N}=2$

In this case, when Anchors are installed, we must ensure that the Tag is on the same side of the two stations attachment. As shown in Fig.2, it is a 3D location map of 2 Anchors. The coordinates of the Anchor, $P_{1}$ and $P_{2}$ is $\left(x_{i}, y_{i}\right),(i=1,2)$; $T$ is the Tag. Calculating two possible Tag coordinates according to distances between Tag and these two Anchors, set as $T_{a}$ and $T_{b}$, their coordinates are $\left(x_{t a}, y_{t a}\right)$ and $\left(x_{t b}, y_{t b}\right)$ respectively.

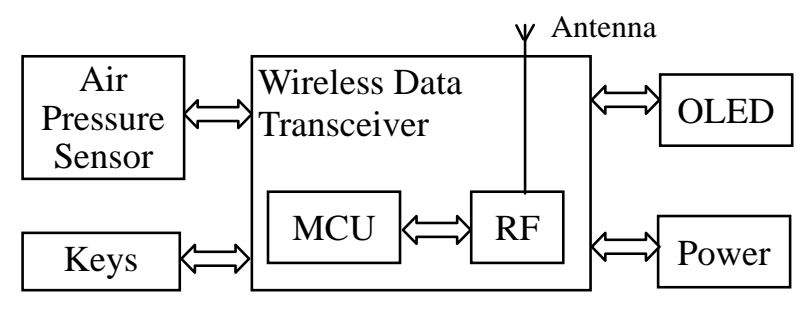

Fig.1. Typical Tag hardware block diagram

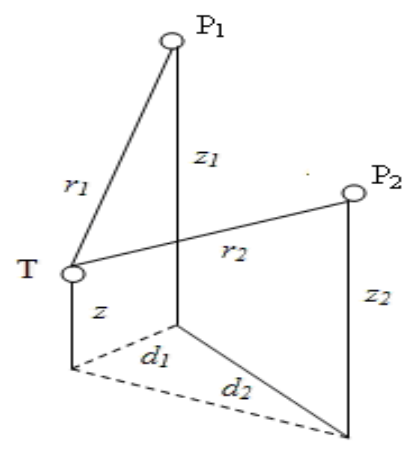

Fig.2. 3D location diagram of two Anchors

In the first possible case, angle between $d_{1}$ and $\mathrm{x}$-axis is:

$\theta=\alpha-\left(90^{\circ}-\arccos \frac{y_{2}-y_{1}}{d_{1,2}}\right)$

In this case, $y$ coordinate of Tag is as follows:

$y_{t a}=y_{1}+d_{1} \sin \theta$

Then $x$ coordinate of Tag is as follows:

$$
x_{t a}= \begin{cases}x_{1}+d_{1} \cos \theta & \text { if } x_{1}<x_{2} \\ x_{1}-d_{1} \cos \theta & \text { if } x_{1} \geq x_{2}\end{cases}
$$

In the second possible case, angle between $d_{1}$ and $x$-axis is:

$$
\theta=\alpha+90^{\circ}-\arccos \frac{y_{2}-y_{1}}{d_{1,2}}
$$

In this case, $y$ coordinate of Tag is as follows:

$y_{t b}=y_{1}-d_{1} \sin \theta$

Then $x$ coordinate of Tag is as follows: 


$$
x_{t b}= \begin{cases}x_{1}+d_{1} \cos \theta & \text { if } x_{1}<x_{2} \\ x_{1}-d_{1} \cos \theta & \text { if } x_{1} \geq x_{2}\end{cases}
$$

When deploying two Anchors, due to knowing which side the Tag is one of the two stations attachment, the coordinates can be selected correctly.

(2) When $\mathrm{N}=3$

Fig. 3 is the schematic diagram of a $3 \mathrm{D}$ positioning with three Anchors. In three Anchors, two-two combination will get three groups, namely: $\left(P_{1}, P_{2}\right)$ and $\left(P_{1}, P_{3}\right)$ and $\left(P_{2}, P_{3}\right)$. Each group of two Anchors and Tag is similar to that in Fig.2. For a group, through the calculation method of the positioning above (1), we can draw two possible coordinates $\left(x_{t a}, y_{t a}\right)$ and $\left(x_{t b}, y_{t b}\right)$. Then the distance between two coordinates and another Anchor is calculated respectively and the shorter distance of point will be selected as the coordinates for the Tag. Finally, averaging the Tag coordinates of three groups will get the position of the Anchor.

(3) When $\mathrm{N}=4$

Fig.4 is the schematic diagram of a 3D positioning with four Anchors. In four Anchors, two-two combination will get six groups, namely: $\left(P_{1}, P_{2}\right) 、\left(P_{1}, P_{3}\right) 、\left(P_{1}, P_{4}\right) 、\left(P_{2}, P_{3}\right) 、\left(P_{2}, P_{4}\right) 、\left(P_{3}, P_{4}\right)$. Each group of two Anchors and a Tag is similar to that in Fig.2. For a group, through the calculation method of the positioning above (1), we can get two possible coordinates $\left(x_{t a}, y_{t a}\right)$ and $\left(x_{t a}, y_{t b}\right)$. Then the sum of the distance between two coordinates and two other Anchors is calculated respectively, the shorter sum of the point will be selected as the coordinates for the Tag. Finally, averaging the Tag coordinates of six groups will get the position of the Tag.

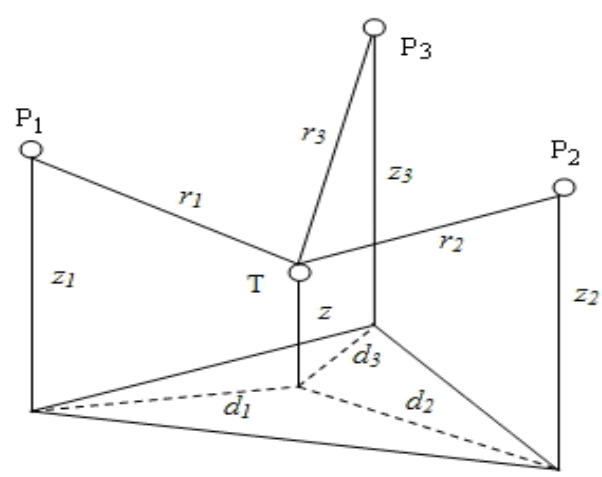

Fig.3. 3D location diagram of three Anchors

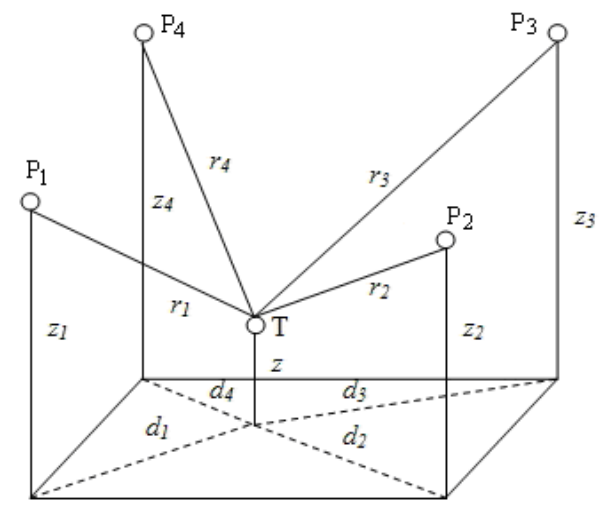

Fig.4. 3D location diagram of four Anchors

\section{Kalman Filter to Eliminate Mutate Values.}

Due to the continuity of actual object's movement path and no paroxysmal activity, measured values should also be continuous and not paroxysmal. At two adjacent times, when the difference of the estimated values exceeds a certain threshold, it means the measured value of the error is too large. For example, at fire scene the sundries indoor (such as iron door) block Tag through direct path in the process of movement from communicating with Anchor by radio waves in a short time, as a result of that, the position of the Tag will change suddenly. Before and after the position's mutations is unpredictable and not related to time, so it can be considered as an error caused by Gaussian noise in measuring process.

Steps of eliminating mutating value using Kalman filter are as follows:

First, calculate the horizontal distance between location position of this moment and last moment $d(k)$, and the altitude difference $h(k)$ :

$$
\begin{aligned}
& d(k)=\sqrt{(x(k)-x(k-1))^{2}+(y(k)-y(k-1))^{2}} \\
& h(k)=|z(k)-z(k-1)|
\end{aligned}
$$

Second, calculate the horizontal movement speed of Tag relative to last moment $v(k)$ and altitude movement speed $u(k)$ :

$$
\begin{aligned}
& v(k)=d(k) /(t(k)-t(k-1)) \\
& u(k)=h(k) /(t(k)-t(k-1))
\end{aligned}
$$


Third, if $v(k)>v_{\max }$ or $u(k)>u_{\max }$, this coordinate value is mutate value, then correct it:

$$
\begin{aligned}
& x(k)=\frac{x^{\prime}(k)-x(k-1)}{v(k-1)} \times v_{\max }+x(k-1) \\
& y(k)=\frac{y^{\prime}(k)-y(k-1)}{v(k-1)} \times v_{\max }+y(k-1) \\
& z(k)=\frac{z^{\prime}(k)-z(k-1)}{u(k-1)} \times u_{\max }+z(k-1)
\end{aligned}
$$

Among them, $x^{\prime}(k), y^{\prime}(k), z^{\prime}(k)$ are respectively the actual measured values at time $k$.

The $x$ coordinates waveform before and after Kalman filter are shown in Fig.5. It is seen that the waveform after Kalman filter is smoother; it improves the positioning accuracy effectively.

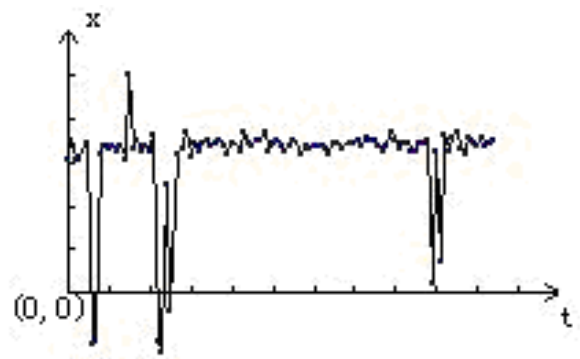

(a) $x$ coordinate waveform before Kalman

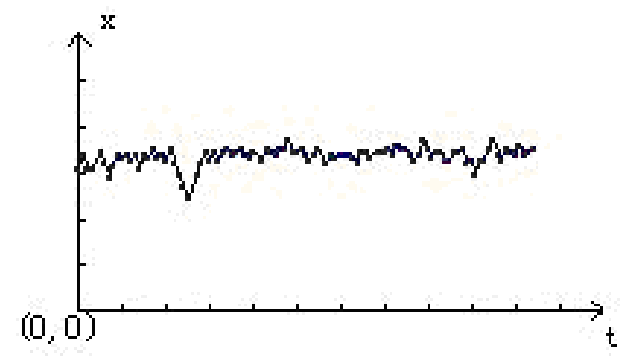

(b) $x$ coordinate waveform after Kalman

Fig.5. x coordinate waveform before and after Kalman

\section{The Results and Analysis of Positioning Experiments}

\section{System Implementation.}

The Microprocessor of Anchors and Tags adopts STM32F103CB. Wireless Radio frequency chips adopt NA5TR1. The NA5TR1, adopting a ranging method called SDS-TWR. By measuring the signal transmission delay time between different nodes, can calculate the real-time distance between the Anchor and Tag. The NA5TR1 is a wireless transceiver chip launched by Nanotron based on CSS. The working frequency of the chip is $2.4 \mathrm{GHz}$ of unlicensed ISM frequency band $(2.4 \sim 2.4835 \mathrm{GHz})$, using the frequency of $2.44 \mathrm{GHz}$, with the advantages of low power consumption, high precision, strong anti- jamming capability, the long transmission distance, etc, which can be effectively applied to indoor mobile object positioning [7][8]. The NA5TR1 typical minimum application system is shown in Fig.6.

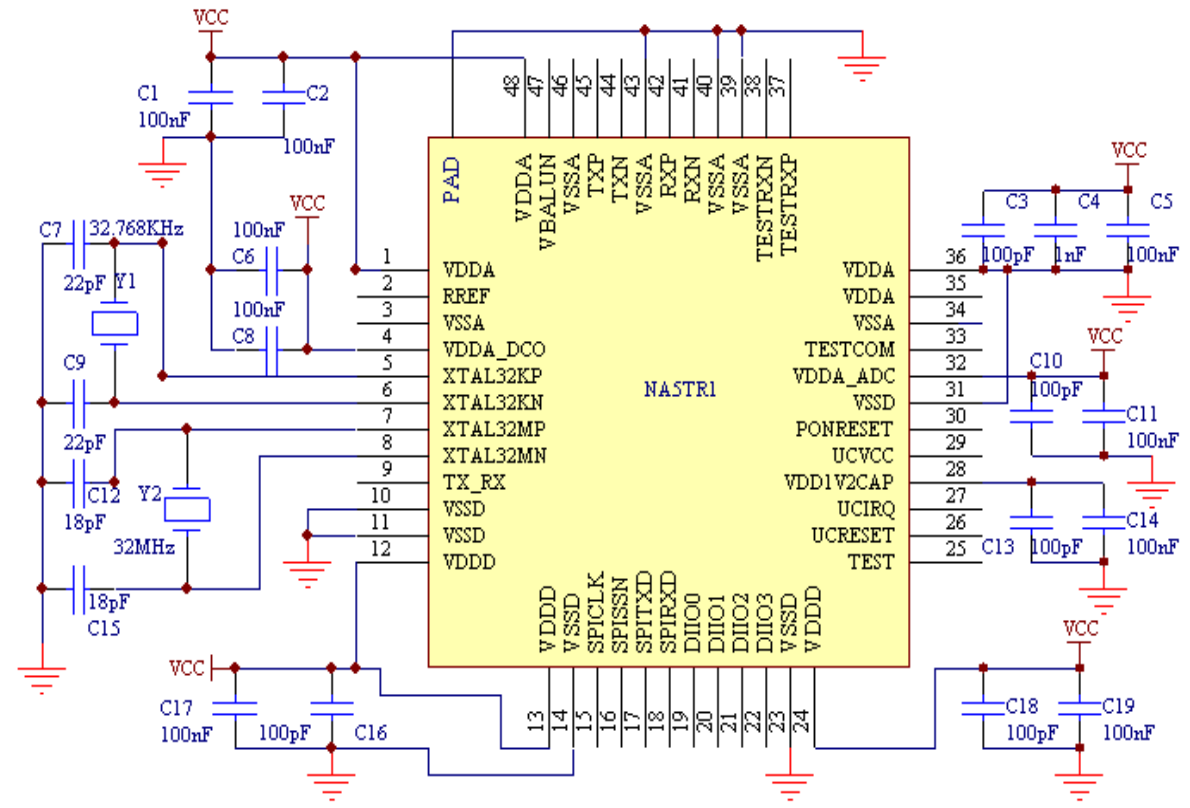

Fig.6. NA5TR1 minimum system 
The pressure sensor adopting BMP085, is used to measure the absolute height of Anchors and Tag [4][6]. The BMP085 digital pressure sensor consists of resistance type pressure sensor, ADC and control units with E2PROM. The control unit is connected to the STM32F103CB through the $\mathrm{I}^{2} \mathrm{C}$.

\section{Experiment Results.}

3D positioning experiment proceeds in the laboratory environment. The coordinates of Anchor 1 , Anchor 2, Anchor 3, Anchor 4 are set as $(0.0,0.0,0.7),(0.0,6.6,0.8),(7.7,6.6,1.3),(7.7,0.0,0.8)$ respectively, and the Tag is placed to the position of $(4.7,3.8,0.7)$. The result of the experiment is shown in Fig.7.

Positioning error is shown in Fig.8. The error represents the distance between measured position and real position of the Tag. In 10 tests, the max positioning error is $0.63 \mathrm{~m}$, the minimum error is $0.24 \mathrm{~m}$ and the average error is $0.51 \mathrm{~m}$. After several experiments, the positioning error can be controlled within $1 \mathrm{~m}$.

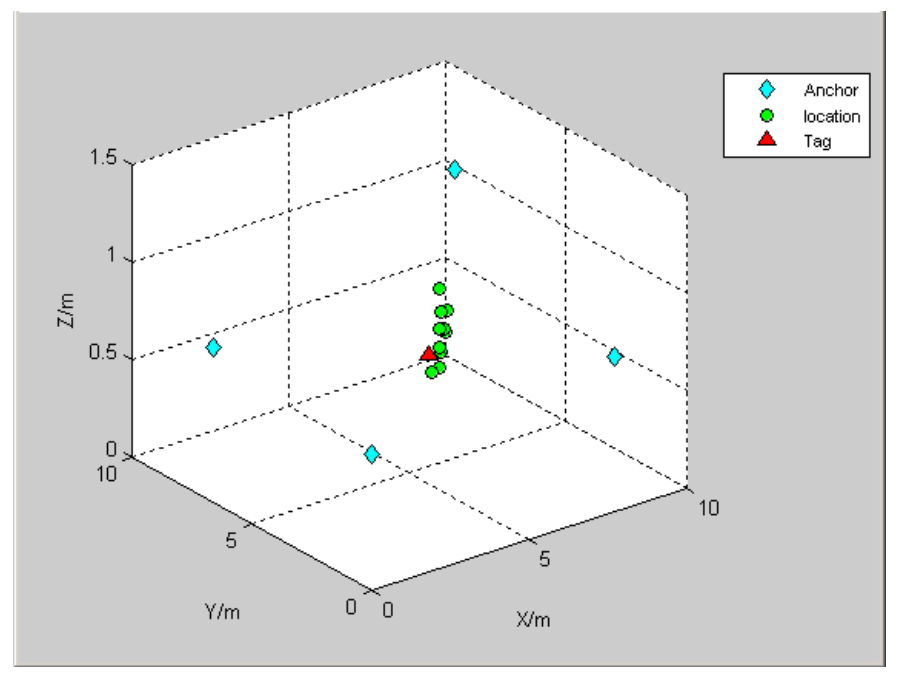

Fig.7. 3D positioning experiment results

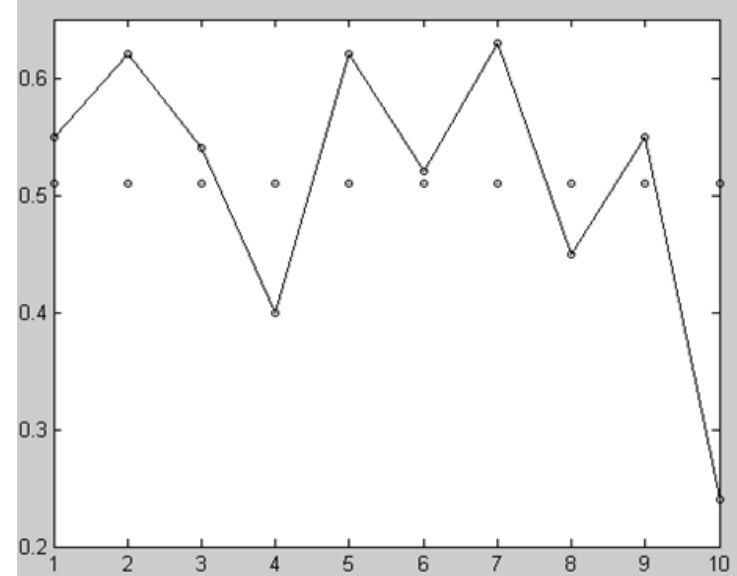

Fig.8. Positioning error

\section{Conclusion}

Aiming that firefighters' safety is not guaranteed at the fire scene, this paper focuses on proposing a 3D positioning algorithm, using Kalman filter to eliminate mutative values and improves the positioning accuracy effectively. At last, verified by positioning experiment, the positioning precision can arrive within 1m, which can position trapped firefighters accurately. Therefore researching the technology of emergency rescue of trapped firefighters has far-reaching practical significance.

\section{Acknowledgement}

This work was supported in part by Zhejiang public welfare technology application research project No.2015C33023, Ningbo social development of science and technology research project No.2014C50022.

\section{References}

[1] Christof Röhrig, Member, IAENG, Julian Lategahn, Marcel MÜLLER and Lars TELLE. Global Localization for a Swarm of Autonomous Transport Vehicles Using IEEE 802. 15. 4a CSS, in Proceedings of the International MultiConference of Engineers and Computer Scientist, Hong Kong, Mar. 2012: 14-16.

[2] Hangoo KANG, Geon woong SEO, and Jihong LEE. Error Compensation for CSS-based Localization System, in Proceedings of the World Congress on Engineering and Computer Science, 
San Francisco, USA, Oct. 2009: 20-22.

[3] Zhengwen YANG, Qiang WU and Yongqiang LU, etal. Enhanced ALOHA Algorithm for Chirp Spread Spectrum Positioning, Pervasive Computing and the Networked World, vol. 7719, 2013: 891-903.

[4] Juncai Wang, Fuping Wang and Ruifeng Hou, etc. A portable altitude measurement system based on BMP085 design [J]. Journal of sensors and micro systems, 2011, 30 (12) : 123-125.

[5] Shengzhan Liang, Xuemei Guo and Xiaotian Yu. Based on BMP085 air pressure sensor and the height of the BP algorithm research and implementation of measurement [J]. Journal of sensing technology, 2013, 26(5) : 654-659.

[6] Jinyan Zhang, Gaoping Lu and Ruxiang Yang. Height measurement system based on pressure sensor BMP085 [J]. Micro computer and application, 2014,(6) : 64-67.

[7] Zhanlei Gu, Cheng He and Ruxiang Yang. Wireless location based on NA5TR1 node design [J]. Journal of electronic design engineering, 2011, 12 (9) : 189-192.

[8] Jiachang Gong and Jun Zhang. Nanotron technologies application in the wireless ranging system [J]. 2011, (3) : 56-58. 